\title{
LPS-induced downregulation of microRNA-204/211 upregulates and stabilizes Angiopoietin-1 mRNA in EA.hy926 endothelial cells
}

\author{
YIJUN ZHANG $^{1,2}$, CAIXIA GAN ${ }^{1,2}$, JIANGBO ZHANG $^{1}$ and DONG CHEN ${ }^{1,3}$ \\ ${ }^{1}$ Sun Yat-sen University Cancer Center, State Key Laboratory of Oncology in South China, \\ Collaborative Innovation Center for Cancer Medicine; Departments of ${ }^{2}$ Pathology and ${ }^{3}$ Urology, \\ Sun Yat-sen University Cancer Center, Guangzhou, Guangdong 510060, P.R. China
}

Received December 12, 2016; Accepted August 14, 2017

DOI: $10.3892 / \mathrm{mmr} .2017 .7400$

\begin{abstract}
Angiopoietin-1 (ANG-1), a ligand of the endothelial cell-specific TIE2 surface receptor, acts in a complementary and coordinated manner with vascular endothelial growth factor during the process of angiogenesis. ANG-1 can be used as a clinically informative biomarker of disease severity and outcome in severe sepsis. The epithelium-specific Ets transcription factor 1 can activate ANG-1 transcription in the setting of inflammation; however, relatively little is known about the regulation of ANG-1 by microRNAs (miRs). It was observed that lipopolysaccharide (LPS) significantly increased ANG-1 mRNA and protein expression in EA.hy926 cells. ANG-1 was identified as a potential target gene of miR-204 and miR-211. Overexpression of miR-204/211 partially reversed the LPS-induced ANG-1 expression in EA.hy926 cells. Furthermore, overexpression of miR-204/211 significantly reduced the activity of a luciferase reporter gene containing the wild-type ANG-1 3'-untranslated region (UTR), but did not influence the activity of a luciferase reporter gene containing the ANG-1 3'-UTR with a mutated miR-204/211 binding site, confirming that miR-204/211 can bind to the ANG-1 3'-UTR and post-transcriptionally regulate ANG-1. Additionally, LPS enhanced the stability of ANG-1 mRNA by reducing the abundance of miR-204/211. Overexpression of miR-204/211 reduced the migration of EA.hy926 cells in vitro. The present study demonstrated that ANG-1 is a novel direct target gene of miR-204 and miR-211; in addition, LPS was able to inhibit this effect by reducing the expression of miR-204 and miR-211.
\end{abstract}

Correspondence to: Dr Dong Chen, Department of Urology, Sun Yat-sen University Cancer Center, 651 Dongfeng Road East, Guangzhou, Guangdong 510060, P.R. China

E-mail: chendong1@ sysucc.org.cn

Key words: angiopoietin-1, miR-204/211, lipopolysaccharide, post-transcriptionally regulate

\section{Introduction}

The endothelial tyrosine kinase receptor (TIE2) is required for vascular remodeling and the maintenance of mammalian vessel integrity (1-3). Angiopoietin-1 (ANG-1) is an angiogenic protein which binds and activates the endothelial TIE2 receptor. The ANG-1/TIE2 signaling cascade plays a crucial role in remodeling and maturation/stabilization of the embryonic vasculature $(4,5)$. Both ANG-1 and TIE2 are widely expressed in the adult vasculature, illustrating their importance in the maintenance of vascular homeostasis. The angiogenic growth factors vascular endothelial growth factor (VEGF), basic fibroblast growth factor (bFGF) and ANG-1 are upregulated in endothelial vessels during inflammatory processes such as wound healing, atherosclerosis and arthritis (6-9). ANG-1 and ANG-2 can be used as clinically informative biomarkers of disease severity and outcome in severe sepsis $(10,11)$. Interleukin-I $\beta$ (IL-1 $\beta)$ and tumor necrosis factor $\alpha$ (TNF- $\alpha$ ) can induce the expression of both VEGF and bFGF $(12,13)$, and Brown et al demonstrated that the expression of ANG-1 is also stimulated by TNF- $\alpha$ (14). Recent studies have shown that microRNAs (miRNAs), short 22 nucleotide non-coding RNAs, can modulate gene expression by binding to the 3'-untranslated regions (UTRs) of their target mRNAs via complementary base pairing, to repress translation or direct sequence-specific degradation of the mRNAs. miRNAs are increasingly being proven to play an important function in the control of inflammatory responses, and have also been associated with the proliferation, migration, adhesion, stimuli response and retraction of vascular cells $(15,16)$. The present study was undertaken to investigate whether the expression of ANG-1 is regulated by specific miRNAs during inflammatory processes. Chen et al, have shown that the functional variant in the 3'-UTR of angiopoietin-1 might reduce stroke risk by interfering with the binding efficiency of miR-211 (17). Here, we provide evidence to show that expression of ANG-1 and the migratory ability of EA.hy926 cells are reduced in response to miR-204/211. Additionally, we demonstrate that miR-204/211 are downregulated in EA.hy926 endothelial cells after exposure to lipopolysaccharide (LPS). 


\section{Materials and methods}

Cell culture and LPS exposure. The EA.hy926 endothelial cell line (18), derived from immortalized human umbilical vein endothelial cells (HUVECs), was cultured in DMEM containing 10\% fetal bovine serum (FBS), $2 \mathrm{mM}$ glutamine, HAT (final concentrations of $100 \mathrm{mM}$ hypoxanthine, $0.4 \mathrm{mM}$ aminopterin and $16 \mathrm{mM}$ thymidine) and antibiotics at $37^{\circ} \mathrm{C}$ in a $5 \% \mathrm{CO}_{2}$ atmosphere. The growth media was replaced every 3-5 days. For transfection and LPS exposure experiments, the cells were seeded in 24-well plates and used at $80 \%$ confluence. The cells were stimulated with $1 \mu \mathrm{g} / \mathrm{ml}$ of LPS for different periods of time $(0,1,2,4,6,12,18$ and $24 \mathrm{~h})$, washed once with PBS, and then harvested.

Plasmid construction. Luciferase reporter constructs were generated by introducing the ANG-1 3'-UTR, carrying a putative miR-204/211 binding site, into the vector pGL3 control (Promega, Madison, WI, USA). The 3'-UTR sequence was amplified by PCR using the primers ANG-1-UTR-Sense (5'-CGGGGTACCGCGCAATGTCAG AAGCGATTATG-3') and ANG-1-UTR-Antisense (5'-GGA AGATCTGTAGTTTGAAGCACAGCAAGC-3') to introduce $K p n I$ and $B g l I I$ restriction sites (underlined). The PCR product was cloned into pGL3 control and the resultant plasmid (pGL3C-WT) was used as a template to produce the mutant plasmid pGL3C-MUT. Site-directed mutagenesis of the miR-204/211 binding site was performed using the Quik-Change Site-Directed Mutagenesis kit (Stratagene, Heidelberg, Germany). Specifically, the bases AAA, complementary to UUU in the seed sequence (UUUCCCUU) of miR-204/211, were mutated to TTT; the three base pairs were mutated without introducing or removing other nucleotides in the binding site. All plasmid DNA was purified using the QIAfilter plasmid kit (Qiagen, Hilden, Germany) and confirmed to have the correct sequence by sequencing (Takara, Dalian, China). miR-NC (empty vector), miR-204 (miRBase accession number: MI0000247) and miR-211 (miRBase accession number: MI0000708) overexpressing retroviral vector carrying were constructed as described by Huang et al $(19,20)$.

Luciferase reporter assay. To analyze the role of the ANG-1 mRNA 3'UTR, EA.hy926 cells were seeded into 24-well plates and transfected with $100 \mathrm{ng}$ of pGL3C-WT/pGL3C-Mut or co-transfected with pGL3C-WT/pGL3C-Mut and miR-204/211 overexpressing plasmids. The cells were also co-transfected with the control plasmid pRL-TK Renilla (Promega). At $24 \mathrm{~h}$ after transfection, the firefly and Renilla luciferase activities of the cell lysates were assayed using the Dual Luciferase Reporter Assay system kit (Promega) with a Modulus Luminometer (Turner Biosystems, Sunnyvale, CA, USA). The relative firefly luciferase activities (RLU) were calculated by normalizing for transfection efficiency against Renilla luciferase activity. All data are the mean \pm SD of at least three independent experiments.

Western blot analysis. Cells were lysed using RIPA buffer containing $1 \%$ NP-40, $0.1 \%$ SDS, $10 \mathrm{mM}$ Tris- $\mathrm{HCl}$ (pH 7.4), $1 \mathrm{mM}$ EDTA, $150 \mathrm{mM} \mathrm{NaCl}$ and protease inhibitor cocktail, and then subsequently sonicated. Protein concentrations were determined using the BCA protein assay kit (Pierce, Rockford, IL, USA) according to the manufacturer's protocol. Equal amounts of protein were resolved by SDS-PAGE and immunoblotted using an anti-ANG-1 antibody and secondary antibody, anti-goat IgG-HRP (Santa Cruz Biotechnology, Santa Cruz, CA). The signals were detected using the ECL Advance Western Blotting Detection kit (Amersham Biosciences, Piscataway, NJ, USA); an anti-glyceraldehyde-3-phosphate dehydrogenase (GAPDH) antibody (Santa Cruz Biotechnology, Santa Cruz, CA). was used as a loading control.

Quantitative reverse transcription-polymerase chain reaction. After treatment with LPS or transfection with plasmids, the cells were harvested and total RNA was isolated using the miRVana miRNA Isolation Kit (Ambion, Austin, TX, USA). The expression of miR-204/211 was quantified using quantitative reverse transcription-PCR (qRT-PCR). The specific miR-204/211 RT primer sequence was 5'-GTCGTA TCCAGTGCAGGGTCCGAGGTATTCGCACTGGATACG ACAGGCAW-3' (W=A or T); the PCR primer sequences for miR-204/211 were 5'-GGGCTTCCCTTTGTCATCCT-3' and 5'-GTGCAGGGTCCGAGGT-3'. U6 snRNA was used as an internal control (5'-CGCTTCGGCAGCACATATAC-3' and 5'-CAGGGGCCATGCTAATCTT-3'). The expression of ANG-1 mRNA was quantified by qRT-PCR using the primers sense 5'-TTTCCTCGCTGCCATTCTGACTC-3' and antisense 5'-TATGGATGTCAATGGGGGAGGTT-3'; GAPDH was amplified as an internal control using the primers sense 5'-CAAAGTTGTCATGGATGACC-3' and antisense 5'-CCATGGAGAAGGCTGGGG-3'.

Assay of mRNA stability. ANG-1 mRNA stability was determined by treating EA.hy926 cells with the transcription inhibitor 5,6-dichloro-1- $\beta$-D-ribobenzimidazole (DRB). The cells were treated with $1 \mu \mathrm{g} / \mathrm{ml}$ LPS for different periods of time $(0,1,2,4$ and $6 \mathrm{~h})$ and then treated with $50 \mathrm{uM} \mathrm{DRB}$ for $24 \mathrm{~h}$ to inhibit transcription. Total RNA was collected at the indicated time points, and the expression of ANG-1 mRNA was determined by qPCR and expressed as a percentage of the initial mRNA level after LPS treatment. The half-life of ANG-1 mRNA was calculated using decay curves.

Wound-scratch assay. The wound-scratch assay was used to investigate cell migration. Briefly, EA.hy926 cells in the log phase were seeded in 6-well plates, cultured for $24 \mathrm{~h}$ until approaching 100\% confluence and then transfected with miR-NC, miR-204 or miR-211-expressing retroviruses. At $24 \mathrm{~h}$ post-transfection, the monolayers were wounded using $10 \mu \mathrm{l}$ pipette tips to produce $300 \mu \mathrm{m}$ wide wounds. The unattached cells were washed away with three washes of PBS, and the monolayers were cultured in media containing $0.1 \%$ FBS. The wounds were photographed immediately and $12 \mathrm{~h}$ after wounding by phase-contrast microscopy at 10x magnification, and the distance migrated was calculated.

Statistical analysis. Each experiment was performed at least three times; paired or unpaired Student's t-tests were used for statistical analysis; significant $\mathrm{P}$-values $(\mathrm{P}<0.05)$ are indicated by asterisks in the figures. 


\section{Results}

LPS induces ANG-1 mRNA and protein expression. To confirm whether ANG-1 is upregulated as an inflammatory response, we investigated the regulation of ANG-1 in EA.hy926 endothelial cells. Firstly, we analyzed the levels of ANG-1 mRNA in EA.hy926 cells stimulated with $1 \mu \mathrm{g} / \mathrm{ml}$ LPS for 1, 2, 4, 6, 12,18 and $24 \mathrm{~h}$ by qRT-PCR. We observed a rapid, significant increase in ANG-1 mRNA expression after $6 \mathrm{~h}$ LPS treatment (about 2.2-fold), which was followed by a slight increase over the remainder of the time course (Fig. 1A). Western blotting was used to determine whether LPS-induced ANG-1 mRNA expression was reflected at the protein level. A time-dependent increase in ANG-1 protein expression was observed, with a maximum at $6 \mathrm{~h}$; however, ANG-1 protein expression did not markedly increase after this time (Fig. 1B).

$L P S$ reduces expression of $m i R-204 / 211$. We analyzed the time-course of miR-204/211 expression in response to LPS treatment. Quantitative RT-PCR demonstrated an inverse correlation between the expression of miR-204/211 and ANG-1 mRNA in LPS-treated EA.hy926 cells (Fig. 1A), indicating that miR-204/211 may mediate LPS-induced expression of ANG-1 mRNA.

LPS treatment stabilizes ANG-1 mRNA. The mechanisms which regulate the transcription of ANG-1 have been extensively researched (21), whereas relatively little is known about the post-transcriptional regulation of ANG-1 during inflammation. Thus, we analyzed the post-transcriptional regulation of ANG-1 during LPS stimulation. To determine if LPS increased the steady-state expression level of ANG-1 mRNA by increasing the stability of ANG-1 mRNA, we treated EA.hy926 cells with PBS or LPS for $6 \mathrm{~h}$, the time point which led to the maximal induction of ANG-1 mRNA (Fig. 1A). Then, DRB, an inhibitor of the TFIIH-associated CTD kinase was added to inhibit ongoing transcription. Total RNA was isolated after 0, 1, 2, 4 and $6 \mathrm{~h}$, and the levels of ANG-1 mRNA were quantified by qRT-PCR. ANG-1 mRNA was highly unstable in PBS-treated EA.hy926 cells, with a half-life of less than $2 \mathrm{~h}$ (Fig. 2). However, LPS treatment increased the half-life of ANG-1 mRNA to approximately $3.5 \mathrm{~h}$. Previous studies have demonstrated that LPS induces the transcription of ANG-1 mRNA (22); however, the response to the transcription inhibitor DRB suggested that LPS could contribute to the stabilization of ANG-1 mRNA.

Overexpression of miR-204/211 downregulate ANG-1 expression. As mRNA stability is primarily regulated by the binding of miRNAs to the 3'UTR of mRNAs, we analyzed the 3'UTR sequence of ANG-1 mRNA and found some adenylate uridylate elements (AREs), a sequence typical of instable mRNA transcripts. The bioinformatic programs TargetScan, PicTar and miRanda were used to predict which miRNAs may target the conserved region of the ANG-1 3'UTR, not other genes such as VEGF, bFGF and ANG-2, which indicates that the function of miRNA204/211 on ANG-1 si specific. Several miRNAs, including miR-204/211, miR-1244, miR-153 and miR-448 were identified to potentially bind the 3'UTR of ANG-1 mRNA, and we hypothesized that ANG-1 may
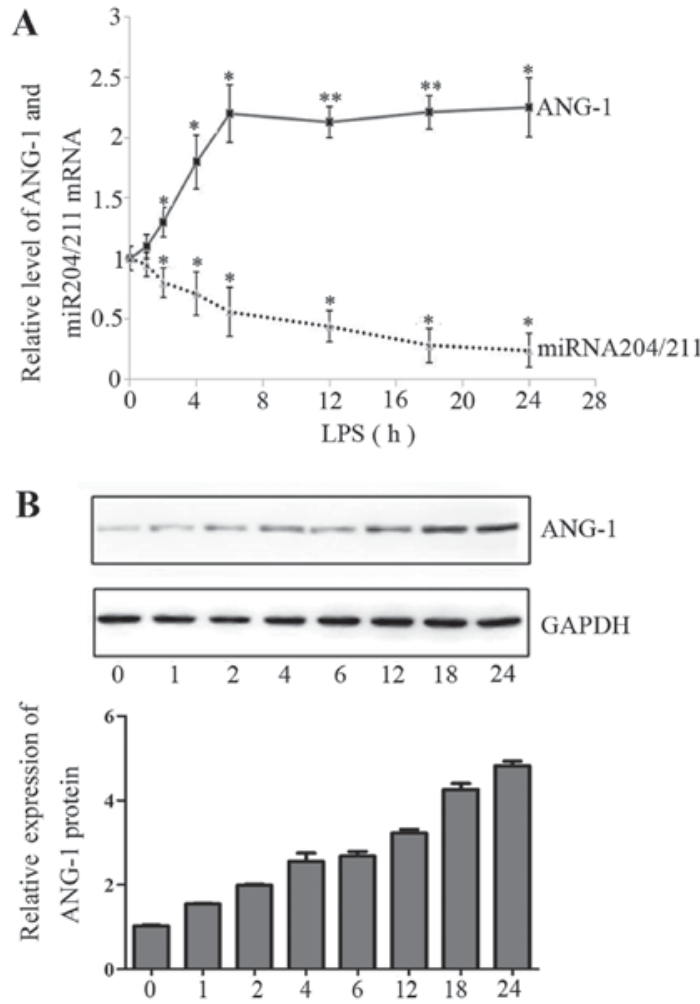

Figure 1. LPS upregulates the expression of ANG-1 mRNA in EA.hy926 cells. EA.hy926 cells were seeded in 24 -well plates, stimulated with $1 \mu \mathrm{g} / \mathrm{ml}$ LPS and harvested at different time points $(0,1,2,4,6,12,18$ and $24 \mathrm{~h})$. (A) Analysis of ANG-1 and miR-204/211 mRNA expression by qPCR. (B) Analysis of ANG-1 protein expression by western blotting. Data are the mean \pm S.E. of three separate experiments; ${ }^{*} \mathrm{P}<0.05 ;{ }^{* *} \mathrm{P}<0.01$, unpaired Student's t-tests.

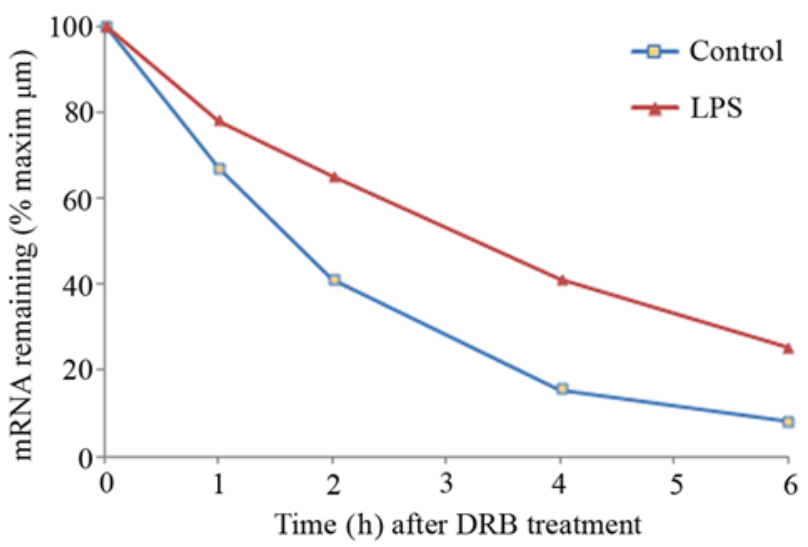

Figure 2. LPS increases the stability of ANG-1 mRNA in EA. hy926 cells EA.hy926 cells were treated with PBS or $1 \mu \mathrm{g} / \mathrm{ml}$ LPS for different time points $(0,1,2,4$ and $6 \mathrm{~h})$ to induce maximal expression of ANG-1 mRNA, and then treated with DRB for $24 \mathrm{~h}$ to inhibit ongoing transcription. The levels of ANG-1 mRNA were determined by qRT-PCR. Data are representative of three experiments.

be regulated by these miRNAs. However, manipulation of miR-1244, miR-153 and miR-448 resulted in no meaningful changes in the cell line following treatment with LPS, therefore it was hypothesized that they were not be involved in the interaction between ANG-1 and miRNA-204/211 MiR-204/211 was singled out for further analysis (Fig. 3A), followed by filtering out those sites that do not appear to be conserved in 
A

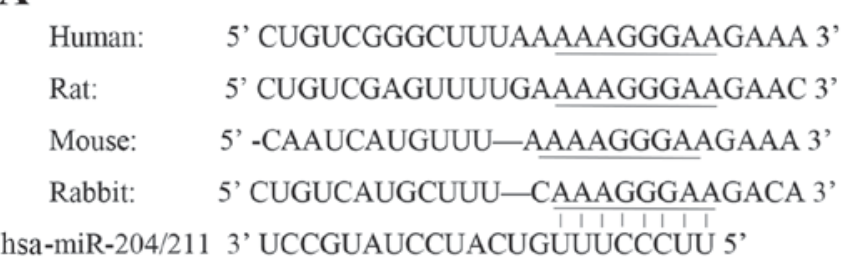

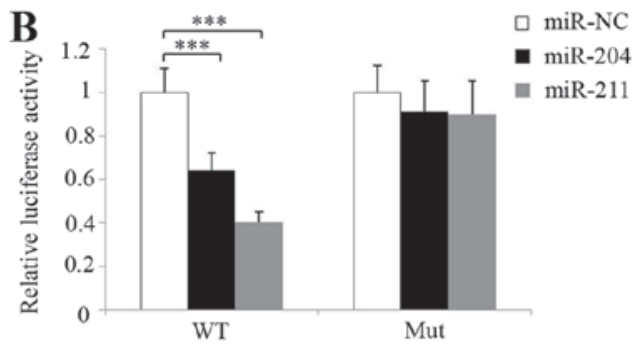

C
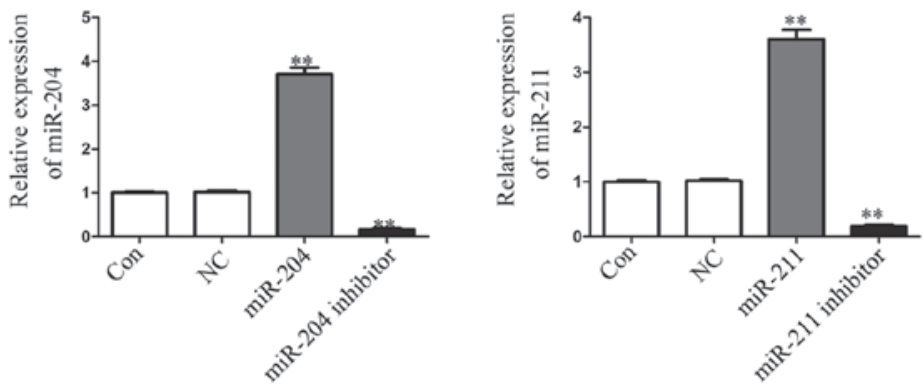

D
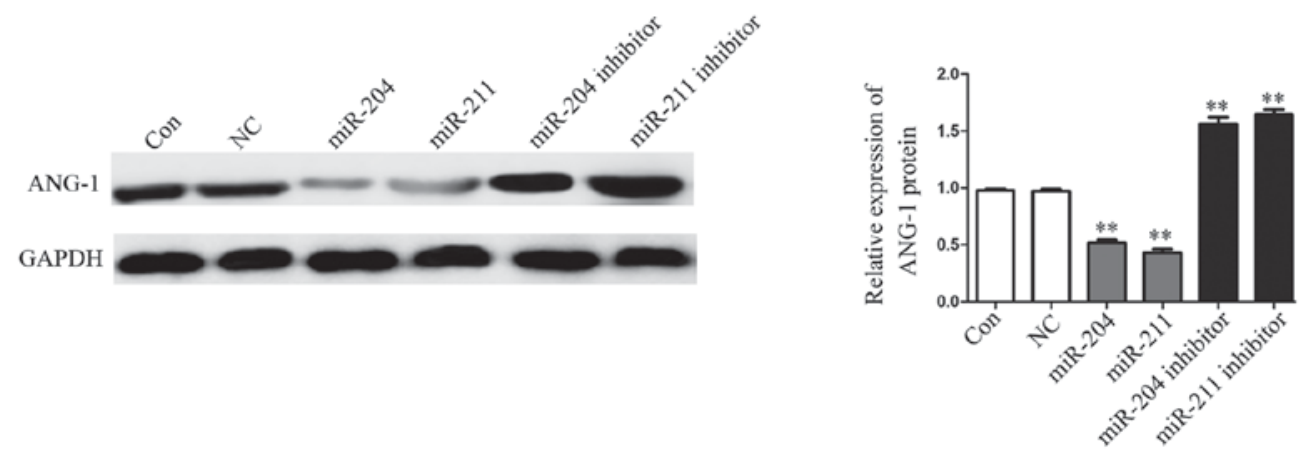

Figure 3. miR-204/211 binds to the 3'UTR of ANG-1 to inhibit the expression of ANG-1. (A) Sequence alignment of miR-204/211 and the ANG-1 mRNA 3'UTR. The miR-204/211 binding sites are underlined, the mutated nucleotides are indicated in bold. EA.hy 926 cells were transfected with luciferase reporter plasmids containing either the wild type (WT) or mutant type (Mut) ANG-1 3'UTR, control (empty vector), miR-204 or miR-211-expressing retroviruses, and the control vector pRL-TK (B) Luciferase reporter gene activity was measured $24 \mathrm{~h}$ after transfection and normalized for transfection efficiency using Renilla activity. (C) and (D). Quantitative PCR and Western blot analyzed the expression of ANG-1 in EA.hy926 cells were transfected with control (empty vector), miR-204 or miR-211-expressing retroviruses. Data are the mean $\pm \mathrm{S}$.E., $\mathrm{n}=3 ;{ }^{* *} \mathrm{P}<0.01 ;{ }^{* * *} \mathrm{P}<0.001$, unpaired Student's $\mathrm{t}-\mathrm{tests}$.

multiple species with the optimal free energy. A dual-luciferase reporter system was employed to validate whether ANG-1 is a direct target gene of miR-204/211. We cloned a 200 bp region of the ANG-1 3'UTR containing the predicted miRNA target site of miR-204/211 (wild type; pGL3C-WT) or the mutated miR-204/211 target site (mutant, pGL3C-Mut) into the luciferase reporter vector pGL3 control. miR-204 or miR-211 were overexpressed in EA.hy926 cells transiently transfected with pGL3C-WT and pGL3C-Mut, and the relative luciferase activities were measured. Overexpression of miR-204 and miR-211 significantly suppressed the luciferase activity of pGL3C-WT by 36.4 and $60.9 \%$, respectively $(\mathrm{P}<0.001)$, whereas overexpression of miR-204 and miR-211 did not significantly affect the luciferase activity of pGL3C-Mut (Fig. 3B). This illustrated that both miR-204 and miR-211 can directly target the 3'UTR of ANG-1. The effects of miR-204 and miR-211 on the endogenous expression levels of ANG-1 were subsequently examined in EA.hy926 cells. Overexpression of miR-204/211 reduced the expression of ANG-1 mRNA by 49.1 and $66.3 \%$, respectively, and the effect can be reversed by adding inhibitors of miR-204/211 (Fig. 3C). Western blotting indicated that overexpression of miR-204/211 in EA.hy926 cells also reduced ANG-1 protein expression by at least 50\% (Fig. 3D).
OverexpressionofmiR-204/211 partlyreverses the LPS-induced ANG-1 expression. To determine if gain-of-function of miR-204/211 could decrease ANG-1 protein levels induced by LPS, we treated the EA.hy926 cells with PBS or LPS, and then retrovirally over-expressed miR-204/211 gene or control vector. Quantitative RT-PCR and western blotting results demonstrated that LPS induced the expression of ANG-1, while, ANG-1 mRNA (Fig. 4A) and protein levels (Fig. 4B) were significantly reduced in the microRNA overexpressing cells. The results indicated that miR-204/211 could partly reverses the LPS-induced ANG-1 expression.

Overexpression of miR-204/211 reduces the migration of EA.hy926 cells in vitro. As ANG-1 is also implicated in cell migration, we examined the effect of miR-204/211 on the migration of EA.hy926 cells in vitro. miR-204/211 were overexpressed in confluent EA.hy 926 cell monolayers, and a wound was formed by scratching with pipette tip. Cell migration was monitored at different time points by light microscopy. After $12 \mathrm{~h}$, the cells in the miR-NC group had migrated $258 \pm 26 \mu \mathrm{m}$. In contrast, the miR-204/211-expressing cells migrated more slowly $(161 \pm 36$ and $174 \pm 31 \mu \mathrm{m}$, respectively; Fig. 5). This data clearly demonstrated that 


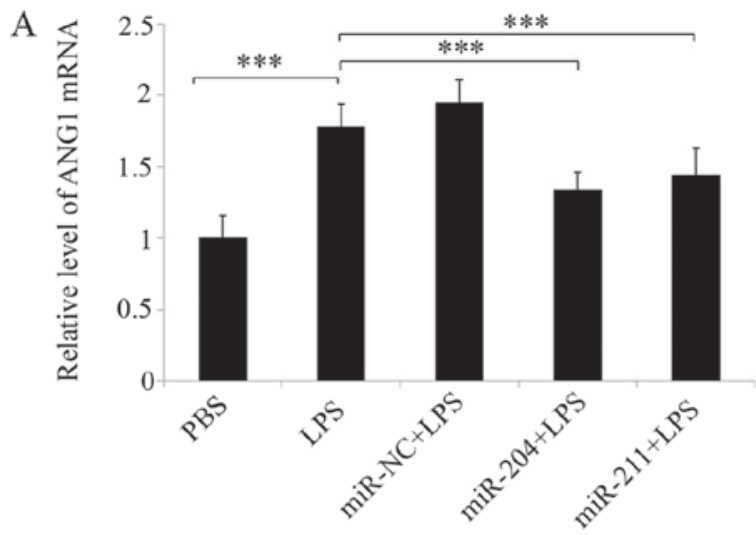

B

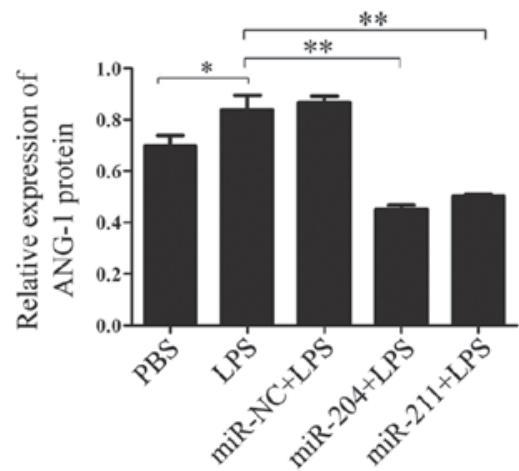

Figure 4. Overexpression of miR-204/211 partly reverses the LPS-induced ANG-1 expression EA.hy926 cells were treated with PBS or LPS (1 $\mu \mathrm{g} / \mathrm{ml})$ for $6 \mathrm{~h}$ following DRB treatment for $24 \mathrm{~h}$ and then infected with control virus or retrovirus expressing miR-204/211. Cells were harvested after $24 \mathrm{~h}$. The expression of ANG-1 was measured by qPCR (A) and Western blotting (B). ${ }^{*} \mathrm{P}<0.05 ;{ }^{* *} \mathrm{P}<0.01 ;{ }^{* * *} \mathrm{P}<0.001$, unpaired Student's t-test, $\mathrm{n}=3$.
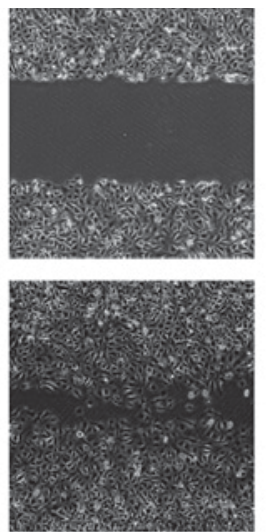

miRNA-NC
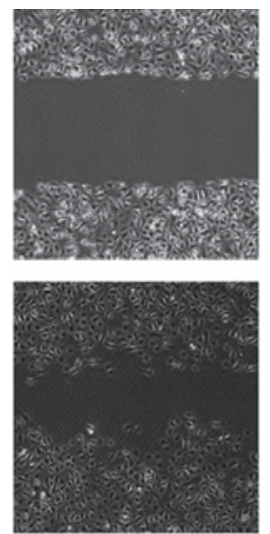

miRNA-204
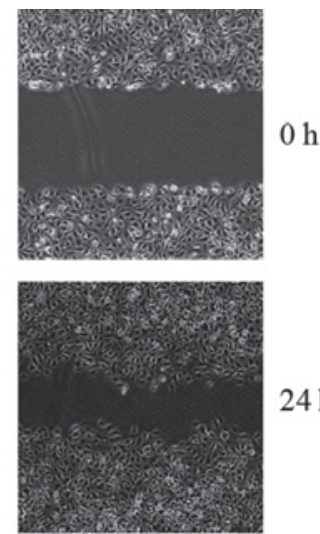

miRNA-211

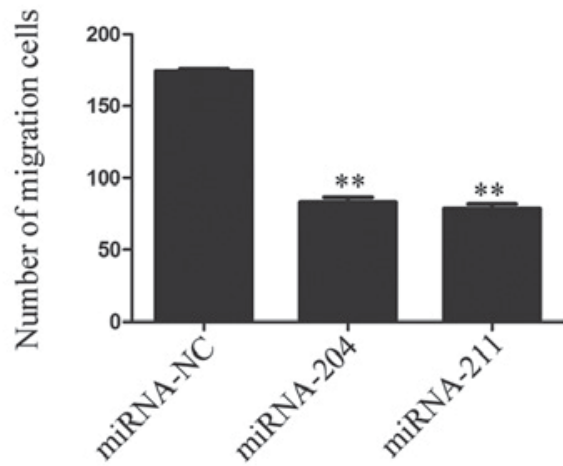

Figure 5. miR-204/211 reduces the migration of EA.hy926 cells in vitro Confluent EA.hy926 cells were transfected with miR-NC (empty vector), miR-204 or miR-211-expressing retroviruses. The cell monolayers were wounded using a plastic pipette tip, the extent of wound closure was monitored by light microscopy; photographs were captured at $12 \mathrm{~h}$. Images representative of three separate experiments are shown. ${ }^{* *} \mathrm{P}<0.01$.

overexpression of miR-204/211 markedly reduced the migration of EA.hy926 cells.

\section{Discussion}

miRNAs are short noncoding RNAs which can regulate a variety of biological processes via regulating the expression of multiple target genes. In the present study, we demonstrated that LPS treatment downregulated the expression of miR-204/211 in EA.hy926 endothelial cells, which upregulated the expression of ANG-1 mRNA. Inflammatory diseases such as rheumatoid arthritis (RA), chronic granulomatous response and certain benign tumors often are accompanied by intense angiogenesis (23-25). During angiogenesis, endothelial cell behavior is modulated by various cytokines. A number of inflammatory cytokines, including TNF- $\alpha$, IL-1 and VEGF are released by inflamed tissues (26). ANG-1 promotes the stabilization of vascular networks and branching morphogenesis, without stimulating endothelial cell proliferation (27). As ANG-1 is a critical mediator of the later stages of blood vessel development and vessel maturation, activation of ANG-1 transcription may also be required during the vascular endothelial cell response associated with inflammatory diseases. Results from studies have shown miRNAs are important regulators of innate immune responses (28). mir-155 is reported to be substantially upregulated in murine macrophages activated with polyriboinosinic:polyribocytid ylic acid [poly (I:C)] or IFN- $\beta$ (29). mir-204 was significantly 
up-regulated in T cells activated by anti-CD3 antibodies in vitro. miR-204 and probably miR-211 are downregulated in inflammation-associated gastric cancer (30). Furthermore, recently data indicated miR-204/211 involved in maintaining epithelial barrier function and cell physiology (31). LPS acts as a classical imitator of gram-negative bacterial infection, which induces the innate and adaptive immune responses and has the capacity to activate endothelial cells (32). In this study, we demonstrated that ANG-1 mRNA expression increased in EA.hy926 endothelial cells exposed to LPS. As post-transcriptional regulation contributes to gene expression during chronic inflammation and cancer (16), we sought to probe the post-transcriptional mechanisms which potentially regulate ANG-1. The transcription inhibitor DRB was used to determine the stability ANG-1 mRNA. After exposure to LPS, the half-life of ANG-1 mRNA increased from 2 to $3.5 \mathrm{~h}$, indicating that ANG-1 mRNA was stabilized in LPS-treated EA.hy926 endothelial cells. We identified a highly conserved 8-mer miR-204/211 binding site in the 3'-UTR of ANG-1 mRNA. miR-204 and miR-211 are closely related homologues. Wang et al reported that miR-204 and miR-211 are highly expressed in human fetal retinal pigment epithelium (hfRPE) cells and are involved in maintaining epithelial barrier function and normal cell physiology (31). miR-204/211 also act as important endogenous negative regulators of runt-related transcription factor 2 (Runx2), which inhibits osteogenesis and promotes adipogenesis of mesenchymal progenitor cells and bone marrow stromal cells (BMSCs) (20). Additionally, the expression of miR-204 is elevated in activated T cells in vitro (33). These results indicate that miR-204/211 are expressed in epithelial cells and may also play a role in inflammatory processes. The use of reporter constructs is an established method to verify potential mRNA destabilization mechanisms. Luciferase assays using an ANG-1 3'UTR reporter gene and point mutation of the miR-204/211 binding sites revealed that miR-204/211 can bind to the ANG-1 3'UTR and post-transcriptionally regulate ANG-1. Furthermore, we showed that LPS treatment could reduce the abundance of miR-204/211 by approximately 40-60\% in EA.hy926 endothelial cells. Therefore, we conclude that LPS negatively modulates miR-204/211, which results in increased expression of ANG-1. ANG-1 has been shown to participate in the endothelial cell migration and proliferation after vascular injury. In the present study, we demonstrate that the overexpression of miR-204/211 dramatically decreased migration of EA.hy926 endothelial cells. These results suggest that downregulation of miR-204/211 may contribute to the repair process of endothelial cell after vascular injury. In the future, further experiments are planned to complete the hypothesis including infecting EA.h926 cells with different amount of miRNA 204/211 retroviral vector, then checking ANG-1 mRNA and protein levels at different timepoints Jennewein et al discovered that the NF-kB-dependent downregulation of peroxisome proliferator-activated receptor 1 (PPAR-1) mRNA expression in response to LPS was due, at least in part, to NF-kB-dependent induction of miR-27b (34). Therefore, it is possible that miR-204/211 may be regulated via the similar NF- $\mathrm{kB}$-dependent mechanism. It is our expectation that the precise mechanisms which regulate ANG-1 will be identified in the near future, which will enable a better understanding of the molecular basis of ANG-1-mediated inflammatory disease.

\section{Acknowledgments}

This study was funded by the Guangdong Natural Science Fund Project, grant number: 2016A030310174.

\section{References}

1. Hansen TM, Singh H, Tahir TA and Brindle NP: Effects of angiopoietins -1 and -2 on the receptor tyrosine kinase tie 2 are differentially regulated at the endothelial cell surface. Cell Signal 22: 527-532, 2010.

2. Augustin HG, Koh GY, Thurston G and Alitalo K: Control of vascular morphogenesis and homeostasis through the angiopoietin-tie system. Nat Rev Mol Cell Biol 10: 165-177, 2009.

3. Chen L, Li J, Wang F, Dai C, Wu F, Liu X, Li T, Glauben R, Zhang Y, Nie G, et al: Tie2 expression on macrophages is required for blood vessel reconstruction and tumor relapse after chemotherapy. Cancer Res 76: 6828-6838, 2016.

4. Erber R, Thurnher A, Katsen AD, Groth G, Kerger H, Hammes HP, Menger MD, Ullrich A and Vajkoczy P: Combined inhibition of VEGF and PDGF signaling enforces tumor vessel regression by interfering with pericyte-mediated endothelial cell survival mechanisms. FASEB J 18: 338-340, 2004.

5. Jackson KA, Majka SM, Wang H, Pocius J, Hartley CJ, Majesky MW, Entman ML, Michael LH, Hirschi KK and Goodell MA: Regeneration of ischemic cardiac muscle and vascular endothelium by adult stem cells. J Clin Invest 107: 1395-1402, 2001.

6. Chen L, Endler A, Uchida K, Horiguchi S, Morizane Y, Iijima O, Toi $\mathrm{M}$ and Shibasaki F: Int6/eif3e silencing promotes functional blood vessel outgrowth and enhances wound healing by upregulating hypoxia-induced factor 2alpha expression. Circulation 122: 910-919, 2010.

7. Abdel-Malak NA, Mofarrahi M, Mayaki D, Khachigian LM and Hussain SN: Early growth response-1 regulates angiopoietin-1-induced endothelial cell proliferation, migration, and differentiation. Arterioscler Thromb Vasc Biol 29: 209-216, 2009.

8. Clavel G, Bessis N, Lemeiter D, Fardellone P, Mejjad O, Ménard JF, Pouplin S, Boumier P, Vittecoq O, Le Loët X and Boissier MC: Angiogenesis markers (VEGF, soluble receptor of VEGF and angiopoietin-1) in very early arthritis and their association with inflammation and joint destruction. Clin Immunol 124: 158-164, 2007.

9. Chan W, Ismail H, Mayaki D, Sanchez V, Tiedemann K, Davis EC and Hussain SN: Fibulin-5 regulates angiopoietin-1/tie-2 receptor signaling in endothelial cells. PLoS One 11: e0156994, 2016.

10. Conroy AL, Phiri H, Hawkes M, Glover S, Mallewa M, Seydel KB, Taylor TE, Molyneux ME and Kain KC: Endothelium-based biomarkers are associated with cerebral malaria in malawian children: A retrospective case-control study. PLoS One 5: e15291, 2010.

11. Paulus P, Jennewein $\mathrm{C}$ and Zacharowski K: Biomarkers of endothelial dysfunction: Can they help us deciphering systemic inflammation and sepsis? Biomarkers 16 (Suppl 1): S11-S21, 2011.

12. Ben-Av P, Crofford LJ, Wilder RL and Hla T: Induction of vascular endothelial growth factor expression in synovial fibroblasts by prostaglandin e and interleukin-1: A potential mechanism for inflammatory angiogenesis. FEBS Lett 372: 83-87, 1995.

13. Paleolog EM, Young S, Stark AC, McCloskey RV, Feldmann M and Maini RN: Modulation of angiogenic vascular endothelial growth factor by tumor necrosis factor alpha and interleukin-1 in rheumatoid arthritis. Arthritis Rheum 41: 1258-1265, 1998.

14. Brown C, Gaspar J, Pettit A, Lee R, Gu X, Wang H, Manning C, Voland C, Goldring SR, Goldring MB, et al: Ese-1 is a novel transcriptional mediator of angiopoietin-1 expression in the setting of inflammation. J Biol Chem 279: 12794-12803, 2004.

15. Lu M, Zhang Q, Deng M, Miao J, Guo Y, Gao W and Cui Q: An analysis of human microRNA and disease associations. PLoS One 3: e3420, 2008. 
16. Khabar KS: Post-transcriptional control during chronic inflammation and cancer: A focus on AU-rich elements. Cell Mol Life Sci 67: 2937-2955, 2010.

17. Chen J, Yang T, Yu H, Sun K, Shi Y, Song W, Bai Y, Wang X, Lou K, Song Y, et al: A functional variant in the 3'-UTR of angiopoietin-1 might reduce stroke risk by interfering with the binding efficiency of microRNA 211. Hum Mol Genet 19: 2524-2533, 2010.

18. Edgell CJ, McDonald CC and Graham JB: Permanent cell line expressing human factor VIII-related antigen established by hybridization. Proc Natl Acad Sci USA 80: 3734-3737, 1983.

19. Kitamura T, Koshino Y, Shibata F, Oki T, Nakajima H, Nosaka T and Kumagai H: Retrovirus-mediated gene transfer and expression cloning: Powerful tools in functional genomics. Exp Hematol 31: 1007-1014, 2003.

20. Huang J, Zhao L, Xing L and Chen D: MicroRNA-204 regulates Runx 2 protein expression and mesenchymal progenitor cell differentiation. Stem Cells 28: 357-364, 2010.

21. Qin J, Chen X, Xie X, Tsai MJ and Tsai SY: COUP-TFII regulates tumor growth and metastasis by modulating tumor angiogenesis. Proc Natl Acad Sci USA 107: 3687-3692, 2010.

22. Mofarrahi M, Nouh T, Qureshi S, Guillot L, Mayaki D and Hussain SN: Regulation of angiopoietin expression by bacterial lipopolysaccharide. Am J Physiol Lung Cell Mol Physiol 294 L955-L963, 2008.

23. Bodolay E, Koch AE, Kim J, Szegedi G and Szekanecz Z: Angiogenesis and chemokines in rheumatoid arthritis and other systemic inflammatory rheumatic diseases. J Cell Mol Med 6 : 357-376, 2002.

24. Szekanecz Z, Pakozdi A, Szentpetery A, Besenyei T and Koch AE: Chemokines and angiogenesis in rheumatoid arthritis. Front Biosci (Elite Ed) 1: 44-51, 2009.

25. Santos IC, Silbiger VN, Higuchi DA, Gomes MA, Barcelos LS, Teixeira MM, Lopes MT, Cardoso VN, Lima MP, Araujo RC, et al: Angiostatic activity of human plasminogen fragments is highly dependent on glycosylation. Cancer Sci 101: 453-459, 2010.
26. Jackson JR, Bolognese B, Kircher CH, Marshall LA, Winkler JD: Modulation of angiogenesis in a model of chronic inflammation. Inflamm Res 46 (Suppl 2): S129-S130, 1997.

27. Papapetropoulos A, Fulton D, Mahboubi K, Kalb RG, O'Connor DS, Li F, Altieri DC and Sessa WC: Angiopoietin-1 inhibits endothelial cell apoptosis via the Akt/survivin pathway. J Biol Chem 275: 9102-9105, 2000.

28. Taganov KD, Boldin MP and Baltimore D: MicroRNAs and immunity: Tiny players in a big field. Immunity 26 : 133-137, 2007.

29. O'Connell RM, Taganov KD, Boldin MP, Cheng G and Baltimore D: MicroRNA-155 is induced during the macrophage inflammatory response. Proc Natl Acad Sci USA 104: 1604-1609, 2007.

30. Lam EK, Wang X, Shin VY, Zhang S, Morrison H, Sun J, Ng EK, Yu J and Jin H: A microRNA contribution to aberrant Ras activation in gastric cancer. Am J Transl Res 3: 209-218, 2011.

31. Wang FE, Zhang C, Maminishkis A, Dong L, Zhi C, Li R, Zhao J, Majerciak V, Gaur AB, Chen S and Miller SS: MicroRNA-204/211 alters epithelial physiology. FASEB J 24: 1552-1571, 2010.

32. Pioli PA, Weaver LK, Schaefer TM, Wright JA, Wira CR and Guyre PM: Lipopolysaccharide-induced IL-1 beta production by human uterine macrophages up-regulates uterine epithelial cell expression of human beta-defensin 2. J Immunol 176: 6647-6655, 2006.

33. Pedersen I and David M: MicroRNAs in the immune response. Cytokine 43: 391-394, 2008.

34. Jennewein C, von Knethen A, Schmid T and Brune B: MicroRNA-27b contributes to lipopolysaccharide-mediated peroxisome proliferator-activated receptor gamma (PPARgamma) mRNA destabilization. J Biol Chem 285: 11846-11853, 2010 